\title{
Preparation and Characterization of Dinitro-ortho-cresol molecularly imprinted Polymer
}

\author{
Raden Tina Rosmalina ${ }^{1}$, Muhammad Bachri Amran², Sri Priatni ${ }^{1}$ \\ ${ }^{1}$ Research Unit for Clean Technology, Indonesian Institute of Sciences, Indonesia 2 \\ Department of Chemistry, Bandung Institute of Technology, Indonesia
}

\begin{abstract}
.
Dinitro-ortho-cresol (DNOC) is a pesticide compound commonly used as herbicides, larvacides, and insecticides in agriculture. DNOC is a toxic compound when exposed to humans and it can cause nausea, stomach disorders, anxiety, excessive burning, etc. It is of great significance to remove DNOC from the environment. In this study, the effective method for the preparation of imprinted by molecular system has been carried out. The benefit of this method is easy for preparation, stable, repeatable use, high mechanical strength, and resistance to heat and pressure. The objective of this research is to synthesize and characterize the functional material (MIP) that can be used for sorbent material of solid phase extraction (SPE). MIP for DNOC was prepared using methacrylic acid (MAA) as a functional monomer, ethylene glycol dimethacrylate (EGDMA) as a crosslinker, benzoyl peroxide (BPO) as an initiator, and acetonitrile as a porogen. The morphous, composition, structure and performance of polymer adsorbent was characterized by FT-IR, SEM, and TGA. The results shows the polymer adsorbent was prepared, successfully. This material had a good binding properties and less waste so it was accordance with the concept of green analytical chemistry. The material was suggested for application in separation of DNOC from other compounds in environmental matrix sample.
\end{abstract}

Keywords : adsorption; DNOC; EGDMA; MIP; MAA

\section{Introduction}

Dinitro-ortho-cresol (DNOC) is one of the compounds commonly used as larvicides, ovisides, and insecticides in agriculture. In the chemical industry, this compound also used as a polymerization inhibitor and as an intermediate compound. In the field of agriculture dinitroortho-cresol usually formulated as an emulsion concentrate in water or oil media. Dinitro-orthocresol is a poisonous compound which when exposed to humans can cause nausea, stomach disorders, anxiety, excessive burning feelings and others. Based on the hazard level, the presence of these compounds in food must be identified and analyzed (Jack K, 2015). Some of the methods that have been widely used for the preparation of dinitro-ortho-cresol samples including liquid-liquid extraction 
(Ferreira et al., 2012) and QuEChERS (Gao et al., 2012). Both of these method has advantages and disadvantages.

The method that will be used in this research is the solid phase extraction method using molecular imprinted polymers (MIP) as solid sorbents. This method can be used for the preparation of dinitroortho-cresol in food samples. Method was chosen because of its low cost, simple to used, stable preparation, repeated use without losing its activity, high mechanical strength, and resistance to heat and pressure. MIP for dinitro-ortho-cresol is prepared using methacrylic acid (MAA) as a functional monomer, ethylene glycol dimethacrylate (EGDMA) as a crosslinker, benzoyl peroxide (BPO) as an initiator, and acetonitril as a porogen. The strength of the interaction between the mold molecule and monomer was evaluated using a UV-VIS spectrophotometer.

Figure 1: MIP formation scheme (Durovi \& Dor, 2015)

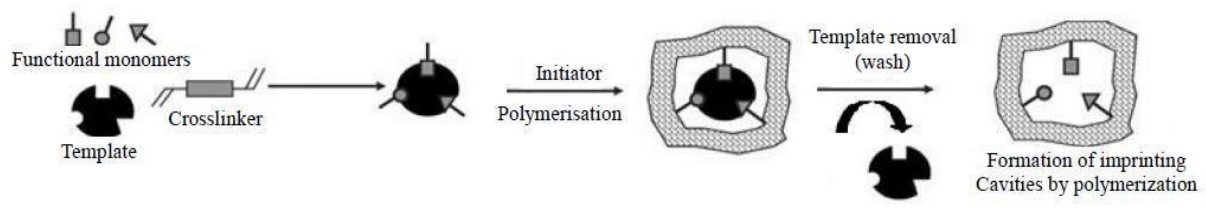

MIP is a polymeric material synthesized by the copolymerization of a print molecule as a target analyte (or analogue) with a functional monomer through covalent or non-covalent interactions with crosslinkers for the appropriate porogen solvent. After the imprinted molecule was removed from the polymer, a three-dimensional space formed which is complementary to the printed molecule (Branger et al., 2013). MIP formation scheme can be seen at Figure 1 (Durovi \& Dor, 2015).

The objective of this research is to synthesize and characterize a functional material in the form of MIP for dinitro-ortho-cresol.

\section{Methods and Discussion}

\subsection{Chemical and Instrument}

Fourier Transform Infra Red Spectrophotometer (FTIR Spectrophotometer) (Shimadzu IR prestige-21), Spectrofotometer UV-VIS Agilent 8453, HPLC UV Agilent 1287 infinity, TGA (JEOL), and Scanning Electron Microscope (SEM) (JEOL-JSM-6510LV). Methanol, acetic acid, acetonitrile, methacrylic acid (MAA), Ethylene Glycol Dimethacrylate (EGDMA), and benzoyl peroxide (BPO) from Merck. Dinitro-ortho-cresol from Sigma aldrich and aquadest. All other chemicals were analytical grade.

\subsection{MIP Synthesis}

Dinitro-ortho-cresol and methacrylic acid (MAA) 1: 1 were put into a glass bottle and dissolved with acetonitrile and stirred for 1 hour. Then crosslinked EGDMA and BPO as an initiator were added. The reaction mixture is shaken and flowed with $\mathrm{N}_{2}$ gas for 10 minutes to remove oxygen, and heated in an oven at $70^{\circ} \mathrm{C}$ for 8 hours. The formed material (MIP) was washed with methanol 
to remove excess reagents. The imprinted molecule (DNOC) was extracted by soccletation using a mixture of methanol-acetic acid solution 9: 1 until the imprinted molecule was not detected with a UV-Vis spectrometer. The MIP was washed again using methanol several times, and heated in an oven at $80^{\circ} \mathrm{C}$ until dry.

\subsection{MIP Characterization with FTIR}

NIP and MIP polymers were characterized using FTIR (KBr pellets). This method becomes very useful for evaluating the existence of several functional groups that should be owned by the synthesized material. The IR spectrum can also be used to see whether or not a new bond has occurred in the MIP.

\subsection{MIP Characterization with Scanning Electron Microscope (SEM)}

NIP and MIP that have been prepared, surface image was taken using SEM with a magnification of 5000 times.

\subsection{MIP Characterization with Thermal Gravimetric Analyzer (TGA)}

This method was carried out to see the physical and chemical changes of MIP due to the heating process TGA measurements was carried out in the temperature range of $25-500^{\circ} \mathrm{C}$. Mass loss data at a certain temperature can be obtained so that the first derivative curve of mass loss can be calculated.

\section{Result and Disscussion}

\subsection{Synthesis and Polymerization}

Synthesis of NIP and MIP was done by bulk method. In this synthesis, MAA and EGDMA were used as the polymer stucture of MIP. MAA as a monomer was expected to interact with dinitroortho-cresol printing through a hydrogen bond. In MIP synthesis, EGDMA works as a morphological controller of the polymer matrix. Polymer morphology can be in the form of gel, macropore, or micro powder. EGDMA also works to stabilize the binding side of the printed molecule, and provide mechanical stability to the polymer matrix. From the point of view of polymerization, generally a high cross-link ratio is preferred to produce materials with adequate mechanical stability. Therefore, this study was used a high cross-link mole ratio, 4 times the functional monomer. The Oxygen inhibits free radical polymerization, so to maximize the propagation rate and ensure polymer reproducibility, removal of dissolved oxygen from the solution must be done (Rahimi \& Vadi, 2014),(Kushwaha et al., 2014). In this study, the removal of dissolved oxygen was carried out by ultrasonication of the solution at the initial stage of adding substances and nitrogen gas flow after adding the initiator. This experiment used a benzoyl peroxide as an initiator which has a radical formation mechanism that was triggered by heating. The solvent used was expected to dissolve all components needed in MIP synthesis. In this research, acetonitrile has been used as a solvent because of its volatility so that the polymer drying 
process can take place relatively quickly. Acetonitril is an agent that is responsible for the formation of pores from a polymer or referred to as porogen.

The ability of MIP to selectively imprinted molecules is influenced by the formation of complexes between the imprinted molecules and the monomers. Imprinted molecules were very important in the molecular printing process because they direct the position of functional monomers in the polymer matrix (Mu'iz, 2015),(Cheng et al., 2015). In the structure of dinitroortho-cresol there was a hydroxyl group that can act as a hydrogen bond acceptor (base), so that acidic methacrylic acid (MAA) was selected which functions as a hydrogen bond donor. Observations during MIP synthesis show that with the addition of imprinting to the NIP solution mixture, it takes longer to produce MIP. The formed polymer can be observed by the presence of yellow deposits in the glass vial where synthesis occurs. White precipitate of NIP was formed within 4 hours, while for synthesis MIP yellow precipitate is formed within 8 hours. The difference in polymerization time between NIP and MIP is due to the presence of dinitro-orthocresol. Dinitro-ortho-cresol molecules which first interact with MAA monomers during prepolymerization can reduce the speed of the initiator of BPO in the formation of free radicals to form polymers. Therefore the MIP polymerization process is slower than the NIP. The porogen solvent remaining after the synthesis process is no longer needed because the polymerization process has ended. After the polymerization process ends, NIP and MIP were washed with methanol. This washing process aims to remove excess residual acid, residual monomers, and other impurities.

Leaching of the imprint molecule in this study was carried out by the socletation method using 9:1 mixture of methanol: acetic acid solvent. The method of socletation is carried out at temperature of $80^{\circ} \mathrm{C}$ for 24 hours. This method can produce more templates so that dinitro-orthocresol can be trapped in MIP, because the solvent was not saturated so that the solvent that enters the soxhlet was dinitro-ortho-cresol free. Therefore, the equilibrium reaction during extraction always leads to the release of dinitro-ortho-cresol from MIP as a result dinitro-ortho-cresol will be more in the solvent.

In addition, the soccletation method also has several advantages, the extraction process were done with hot solvent that supports dissolution of the print molecule, and no filtration need after extraction to collect MIP particles. Leaching of the print molecule was an important step in the MIP synthesis process. If there were remaining print molecules in the MIP, the cavities available for the binding process of the target molecule will be reduced.

\subsection{MIP Characterization with FTIR}

After the polymerization process, a functional group analysis was performed using FTIR on NIP, MIP, and MIP after leaching. The FTIR spectrum obtained can be seen in Figure 2.

The results above show the difference in the FTIR spectrum between NIP and MIP, there was an additional peak on MIP in the wave number around $1540 \mathrm{~cm}^{-1}$ which indicates the presence of $\mathrm{CN}$ groups. This shows the presence of $\mathrm{C}-\mathrm{N}$ groups that appear on MIP originating from dinitroorthocresol. Otherwise, on NIP and MIP after leaching there was no peak in the wave number $1540 \mathrm{~cm}^{1}$ because it does not contain dinitro-ortho-cresol. 


\section{iCmets}

\section{$2^{\text {nd }}$ International Conference on Modern Research in}

Figure 3 shows that the FTIR spectrum MIP with dinitro-ortho-cresol has the same peak at wave number around $1540 \mathrm{~cm}^{-1}$, this ensures that the peak at wave number $1540 \mathrm{~cm}^{-1}$ really originates from dinitro-ortho-cresol. Moreover, Figure 2 shows that the NIP and MIP stretching vibrations of C-H appear at wave number $2956.87 \mathrm{~cm}^{-1}$. The peaks that appear on NIP, MIP, and MIP after leaching at wave number $1726.29 \mathrm{~cm}^{-1}$ indicate the existence of vibrations of $\mathrm{C}=\mathrm{O}$ carbonyl ester stretches derived from cross polymerization of MAA and EGDMA. Similarly, peaks at NIP, MIP, and MIP after leaching at the wave number $1259.52 \mathrm{~cm}^{-1}$ indicate the existence of C-O stretching vibrations.

Figure 2: FT-IR spectrum of NIP (black), MIP (red) and MIP after leaching (blue)

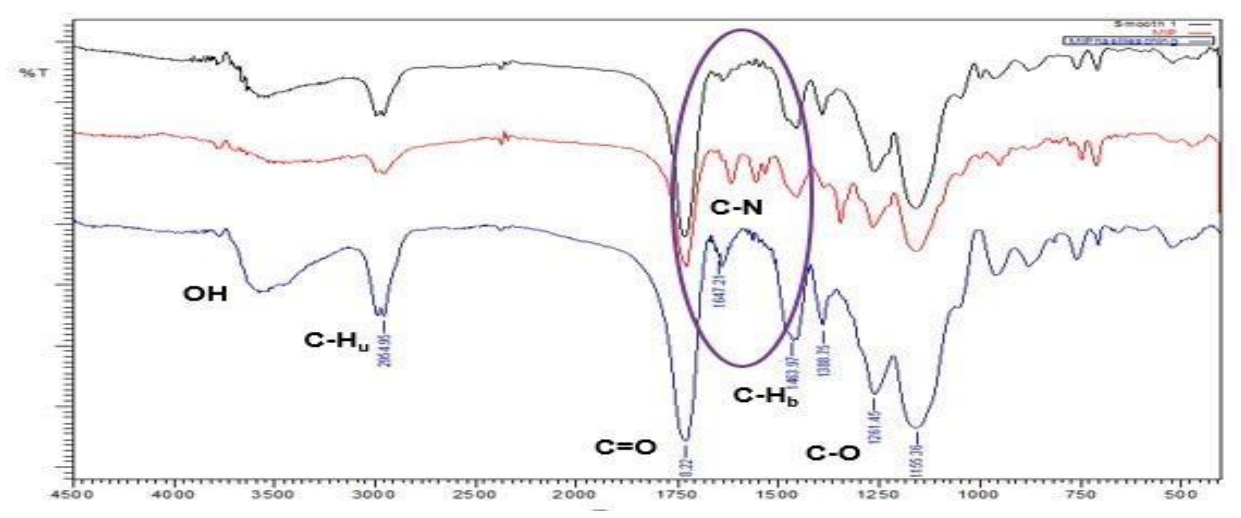

Figure 3: FTIR spectra of MIP (red) and Dinitro-ortho-cresol (black)

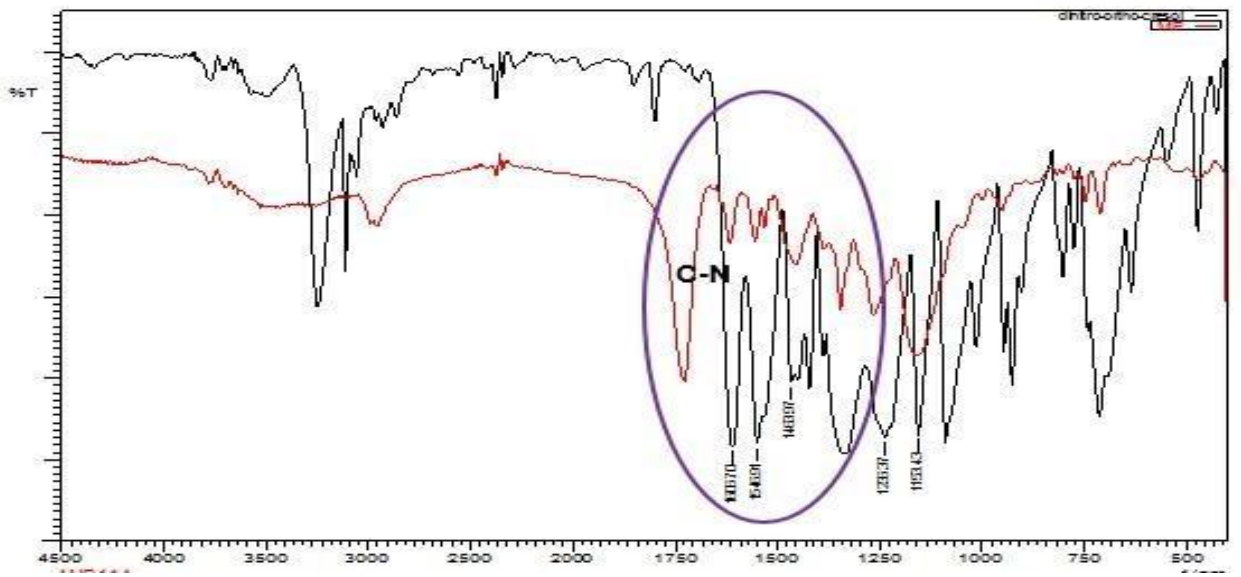

\subsection{MIP Characterization with Scanning Electron Microscope (SEM)}

To see the surface morphology of the NIP and MIP, a surface image was taken with SEM. The results obtained using 5000 times magnification showed differences in surface morphology between NIP, MIP-DNOC, MIP after leaching, and MIP that had been re-contacted with the 


\section{iCmets}

$2^{\text {nd }}$ International Conference on Modern Research in

printed molecule. NIP and MIP have different porosity and surface. Figure 4 (a) shows a NIP surface that looks homogeneous and smooth, whereas in (b) MIP-DNOC the surface looks more heterogeneous and rougher. In Figure (c) MIP after leaching, the surface becomes more homogeneous and smoother, while in Figure (d) MIP was brought back into contact with a dinitroortho-cresol imprinted molecule so that the surface looks more rough and heterogeneous.

Figure 4: Image of surface morphology of SEM results with magnification of 5000 times (a) NIP; (b) MIP-DNOC;

(c) MIP after leaching (d) MIP recontacted with dinitro-ortho-cresol molecular imprinted
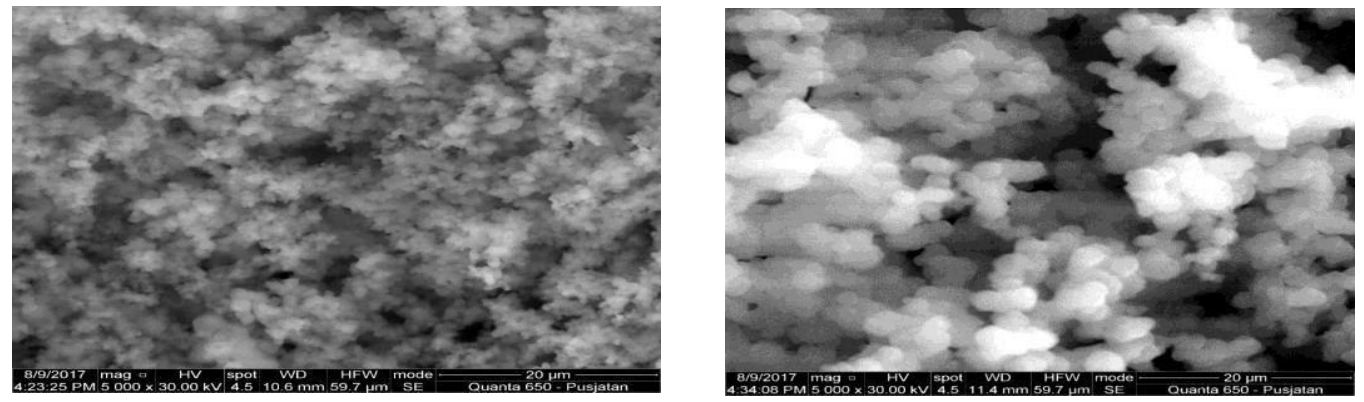

(a)

(b)

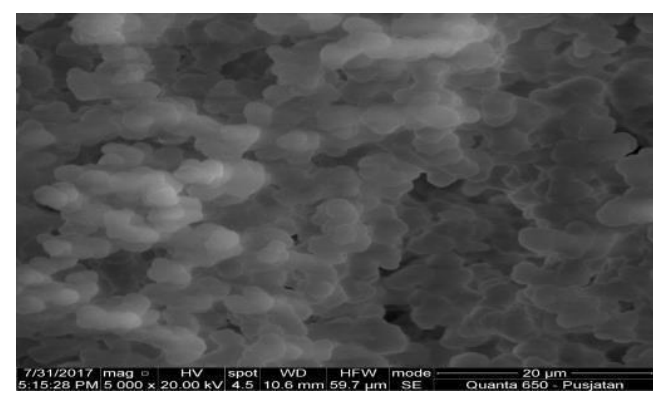

(c)

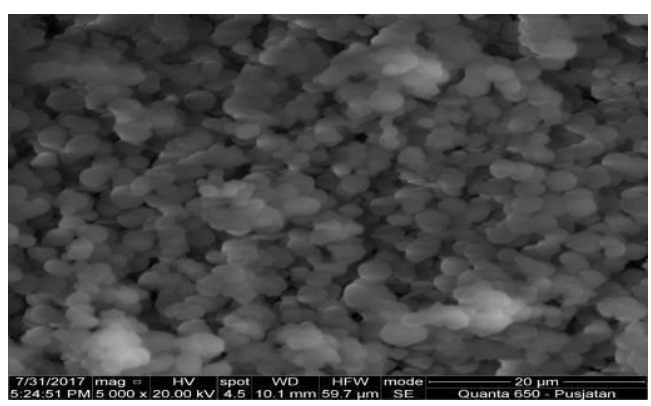

(d)

\subsection{MIP Characterization with Thermal Gravimetric Analyzer (TGA)}

In this study, NIP and MIP were poured into a special container on the TGA instrument, then analyzed to determine the mass of the material which was reduced when heated from room temperature 25 to $500^{\circ} \mathrm{C}$. 


\section{iCmets}

$2^{\text {nd }}$ International Conference on Modern Research in

MUNICH, GERMANY

ENGINEERING, TECHNOLOGY AND SCIENCE

6 - 8 DECEMBER, 2019

Figure 5: TGA Thermograms of NIP and MIP

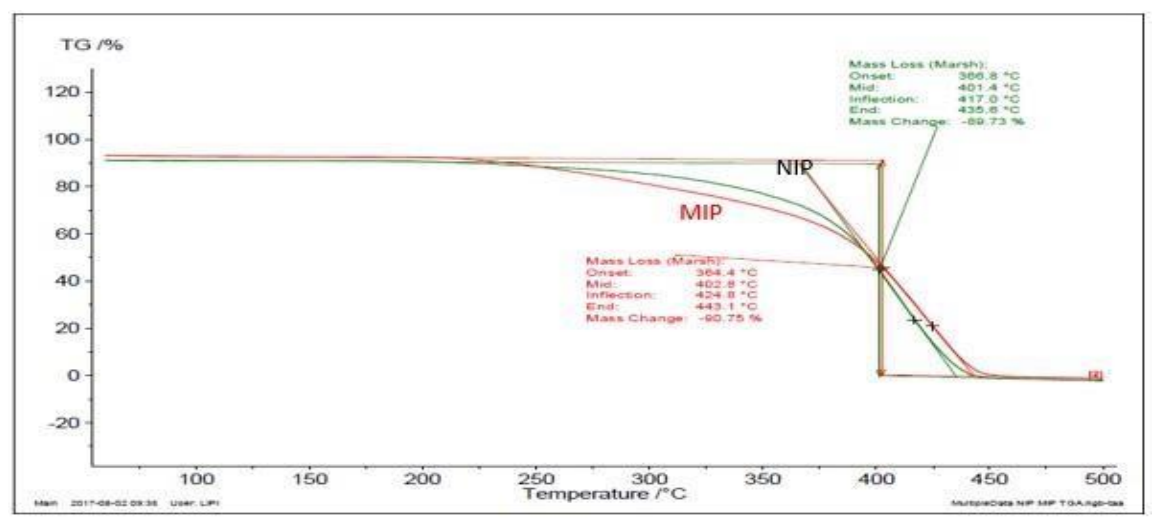

Figure 5 and 6 show that the mass loss that occurs in NIP was only one stage, at $417^{\circ} \mathrm{C}$, while MIP has two stages of mass loss at temperatures around 290 and $424^{\circ} \mathrm{C}$. This was because MIP contains dinitro-ortho-cresol printed molecules. Therefore, the mass loss that occurs in MIP by a temperature of around $290^{\circ} \mathrm{C}$ indicates the mass loss from the dinitro-ortho-cresol molecular imprinted, and this fit to the decomposition temperature of dinitro-ortho-cresol which was $312^{\circ} \mathrm{C}$. Subsequently, there was a shift in the temperature of the mass removal from NIP and MIP in the second stage from 417 to $424^{\circ} \mathrm{C}$ indicating the presence of other compounds in MIP. Overall, MIP experienced greater mass loss than NIP, which was $90.75 \%$, while NIP was only $89.73 \%$.

Figure 6: DTG Thermograms of NIP and MIP

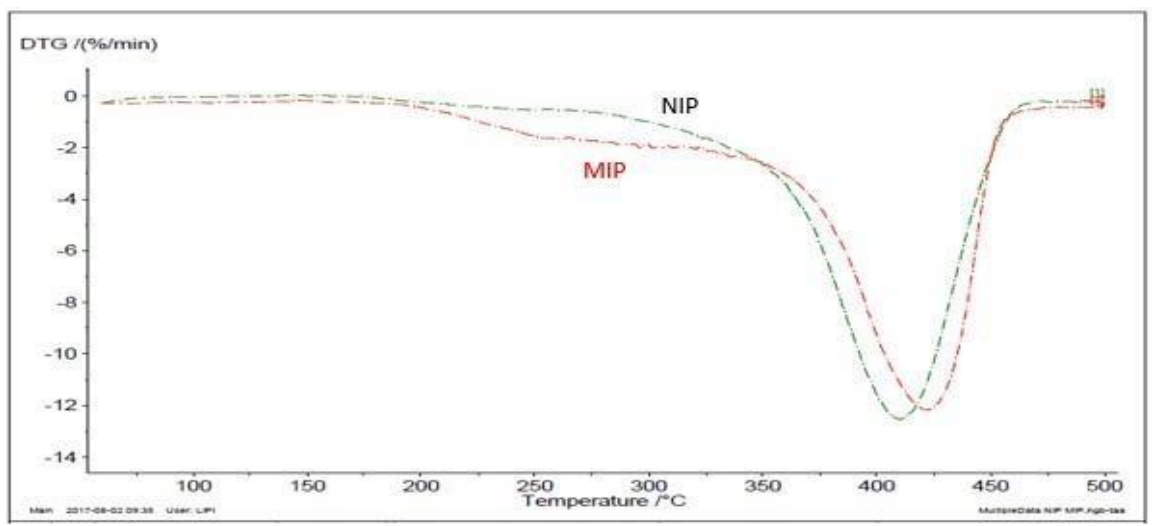




\section{Conclusion}

From the research that has been done, it can be concluded that MIP-DNOC has been successfully synthesized using the bulk method. The results shows in the FTIR spectrum between NIP and MIP, there was an additional peak on MIP in the wave number around $1540 \mathrm{~cm}^{-1}$ which indicates the presence of C-N groups. SEM using 5000 times magnification showed differences in surface morphology between NIP and MIP, the surface of MIP looks more rough and heterogeneous than NIP. The results from characterization with TGA shows that the mass loss that occurs in NIP is only one stage, at $417^{\circ} \mathrm{C}$, while MIP has two stages of mass loss at temperatures around 290 and $424^{\circ} \mathrm{C}$ conform to the decomposition temperature of dinitro-ortho-cresol which was $312^{0} \mathrm{C}$.

\section{Acknowledgment}

The authors are grateful to Ristekdikti which provided the financial support for doing this research.

\section{References}

[1] Jack, K. (2015). A historical perspective and the development of molecular imprinting polymer-A review. Journal of Chemistry International I, vol. 4, pp. 202-210.

[2] Ferreira, I., Fernandes, J. O., \& Cunha, S. C. (2012). Optimization and validation of a method based in a QuEChERS procedure and gas chromatography e mass spectrometry for the determination of multi-mycotoxins in popcorn. Food Control, vol. 27(1), pp. 188- 193.

[3] Gao, N., Dong, J., Liu, M., Ning, B., Cheng, C., Guo, C., \& Zhou, C. (2012). Development of molecularly imprinted polymer films used for detection of profenofos based on a quartz crystal microbalance sensor. Jounal of The Royal Society of Chemistry, pp. 1252-1258.

[4] Đurovi, R., \& Đor, T. (n.d.). (2015). Modern Extraction Techniques for Pesticide Residues Determination in Plant and Soil Samples. Talanta.

[5] Branger, C., Meouche, W., \& Margaillan, A. (2013). Reactive \& Functional Polymers Recent advances on ion-imprinted polymers. Reactive And Functional Polymers. Journal of Elsevier ScienceDirect.

[6] Rahimi, M, dan Vadi, M. (2014) : Langmuir, Freundlich and Temkin Adsorption Isotherms of Propranolol on Multi-Wall Carbon Nanotube. Journal Of Modern Drug Discovery, vol. 5, pp. 1-3.

[7] Kushwaha, A. K,. Gupta, N,. dan Chattopadhyaya, M. C. (2014) : Removal of cationic methylene blue and malachite green dyes from aqueous solution by waste materials of Daucus carota. Journal of Saudi Chemical Society, vol. 18, pp. 200-207.

[8] Mu'iz, F. A. (2015) : Adsorben Berbasis Alginat Terprotonasi Untuk Adsorpsi Ion Ce ${ }^{3+}$. Skripsi Program Sarjana, Institut Teknologi Bandung. 
[9] Cheng, J,. Ding, C,. Li, X,. Zhang, T,. dan Wang, X. (2015) : Rare Earth Element Transfer from Soil to Navel Orange Pulp (Citrus sinensis Osbeck cv. Newhall) and the Effects on Internal Fruit Quality. PLoS ONE, vol. 10, pp. 1-15.

[10] Article, O. (2013). Preparation of molecularly imprinted polymers for heptachlor: An organochlorine pesticide. Journal of Chronicles of Young Scientist, vol. 4(1), pp. 46-50.

[11] K. P. Singh., Ajeet Kumar., Shweta, T., Rakesh, S., \& Prashant, S. (2014). Selective Recognition of Endosulfan Pesticide in Environmental Matrix with Molecularly Imprinted Polymer Membrane. Research Journal of Chemical Sciences, vol. 4(4), pp. 63-70.

[12] Bradley, S., Gosen, V., Philip, L., Verplanck, Keith, R. L., dan Gambogi, J. (2004) : The Rare Earth Elements- Vital to Modern Technologies and Lifestyles. USGS Minereral Resource Program, 2327-6932.

[13] Bahti, H. H., Mulyasih, Y., dan Anggraeni, A., (2011) : Extraction and chromatographic studies on rare-earth elements ( REEs ) from their minerals : the prospect of REEs production in Indonesia?. Proceedings of the 2nd International Seminar on Chemistry, pp. 24-25, 421430.

[14] Christiano, B,. Ferreira, S,. dan Simões, F. (2014) : Application of a new carboxylatefunctionalized sugarcane bagasse for adsorptive removal of crystal violet from aqueous solution: Kinetic, equilibrium and thermodynamic studies. Industry Crops and Product, vol. 65, pp. 521-534.

[15] Chen, X. (2015) : Modeling of Experimental Adsorption Isotherm Data, Information, vol. 6, pp. 14-22. 